\title{
Tratamiento del Colorante Azo Rojo Directo 23 mediante Reactores Discontinuos Secuenciados Anaerobios/Aerobios
}

\author{
Fernanda Morales-Guzmán ${ }^{1}$ y Rosa M. Melgoza-Alemán ${ }^{2}$ \\ Universidad Autónoma del Estado de Morelos, (1) Postgrado en Ingeniería y Ciencias Aplicadas, \\ (2) Facultad de Ciencias Químicas e Ingeniería/Centro de Investigación en Ingeniería y Ciencias \\ Aplicadas, Av. Universidad 1001, Col. Chamilpa, 62209 Cuernavaca, Morelos-México \\ (e-mail: rmelgoza@uaem.mx)
}

\begin{abstract}
Resumen
Se evaluó el tratamiento del colorante azo Rojo Directo 23 mediante un proceso anaerobio/aerobio integrado en un sólo reactor. Se utilizaron dos reactores: uno de lecho expandido con carbón activado granular como mediador redox y otro conteniendo la biomasa suspendida, ambos operados en discontinuo. Se evaluó la eficiencia del proceso mediante la reducción y transformación del colorante a aminas aromáticas totales en la fase anaerobia y la degradación de éstas en la fase aerobia. La eliminación del colorante fue por microorganismos y no por adsorción en el carbón activado granular. Las concentraciones de colorante utilizadas fueron de 25 a $100 \mathrm{mg} \mathrm{L}^{-1}$. En el reactor de lecho expandido la eficiencia de degradación global del colorante fue del $90 \%$ y la eliminación de las aminas formadas fue del $100 \%$.
\end{abstract}

Palabras clave: Azo Rojo Directo 23, colorante, proceso anaerobio/aerobio, aminas aromáticas, carbón activado

\section{Treatment of Direct Red 23 Azo Dye by Anaerobic/Aerobic Sequencing Batch Reactors}

\begin{abstract}
The treatment of the azo dye Direct Red 23 was evaluated by an anaerobic/aerobic process done in a single reactor. Two reactors were used: one was an expanded-bed with granular activated carbon as redox mediator and the other containing the suspended biomass, both operated in batch mode The efficiency of the process was evaluated by the reduction and transformation of the azo dye to total aromatic amines in the anaerobic phase and the degradation of these in the aerobic phase. The elimination of azo dye direct red was by microorganisms and not by adsorption in the granular activated carbon. The concentration of azo dye used was of 25 to $100 \mathrm{mg} \mathrm{L}^{-1}$. In the expanded-bed reactor the removal efficiency of the dye was $90 \%$ and the elimination of the amines formed was of $100 \%$.
\end{abstract}

Keywords: azo Direct Red 23, dye, anaerobic/aerobic process, total aromatic amines, activated carbon 


\section{INTRODUCCIÓN}

Cuando se tratan aguas residuales de procesos textiles, la remoción de colorantes es uno de los principales objetivos del tratamiento. La industria textil representa un importante sector económico a nivel mundial. Con el aumento en la demanda de productos textiles, la industria textil a incrementado los volúmenes de descargas de aguas residuales, siendo este uno de los principales problemas de contaminación en el mundo (Dos Santos et al., 2007). Los colorantes azo son utilizados para el proceso de teñido de textiles y se estima que las cantidades que no se fijan a las fibras dependen de la clase de colorante empleado, variando del $2 \%$ cuando se usan colorantes básicos al $50 \%$ cuando se usan colorantes reactivos (O'Neill et al., 1999); estas cantidades pérdidas de colorante se liberan al ambiente como aguas residuales (Tan et al., 2000). Las descargas de colorantes en el medio ambiente deben evitarse no sólo por razones estéticas, sino también por que muchos colorantes azo y los productos derivados del rompimiento del enlace azo son tóxicos a la vida acuática y mutagénicos a los humanos (Weisburger, 2002). Existen estudios relativos a la degradación y toxicidad de los colorantes azo en condiciones anaerobias y aerobias en sedimentos de cuerpos receptores, donde se realiza la descarga de aguas residuales (Isik y Sponza, 2007; Ekici et al., 2001), sin embargo el conocimiento del destino y la toxicidad de los metabolitos generados es escaso.

Los colorantes azo son compuestos químicos xenobióticos caracterizados por la presencia de uno o más grupos azo (-N=N-), considerados uno de los más importantes grupos de colorantes sintéticos. Son muy recalcitrantes a los procesos biodegradativos debido a su alta estabilidad a la luz y al ataque microbiano, originando una severa contaminación de ríos y agua subterránea en las áreas dónde se localizan industrias textiles y de colorantes (Stolz, 2001). Entre la familia de colorantes azo, los colorantes directos son moléculas relativamente grandes con alta afinidad por las fibras de celulosa, mediante fuerzas de Van der Walls. La mayoría tiene uno o más enlaces azo o compuestos del tipo de ftalocianinas, estilbenos u oxacinas. De acuerdo al Colour Index (C.I., 1992), los colorantes directos forman la segunda más grande clase de colorantes, cerca de 1600 colorantes son enlistados, pero sólo aproximadamente el $30 \%$ son producidos actualmente (van der Zee et al., 2003). El colorante azo Rojo Directo 23 (RD23 C.I. 29160) es uno de los colorantes con mayor aplicación en la industria textil, es utilizado en el teñido de fibras de algodón, lino y rayón; es estable a la luz, se absorbe fácilmente en el agua y su grado de fijación en la fibra va del 70 al 95 \% (O’Neill et al., 1999). En la figura 1 se muestra la estructura del colorante.

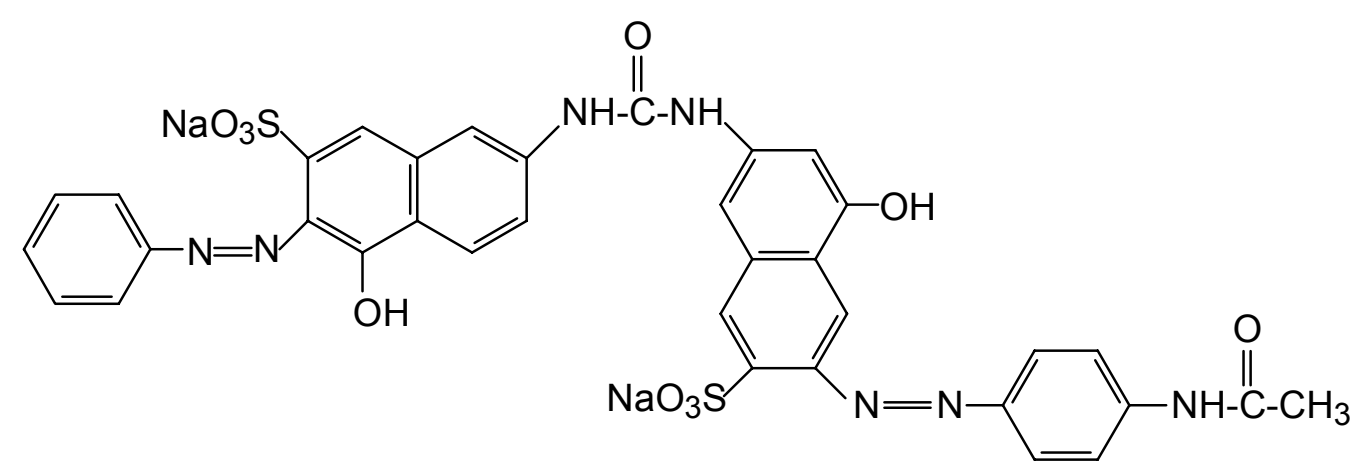

Fig. 1: Estructura química del colorante azo rojo directo 23 (RD23 Cl 29160)

Los colorantes azo son resistentes a la biodegradación aerobia en plantas de tratamiento convencionales (Shaul et al., 1991), sin embargo bajo condiciones anaerobias sufren fisión reductiva produciendo aminas aromáticas incoloras, que generalmente requieren condiciones aerobias para su biodegradación (Ekici et al., 2001). Los procesos anaerobios y aerobios son complementarios, de forma independiente ninguno es suficiente para eliminar la toxicidad de las aguas residuales textiles (Frijters et al., 2006). Algunas aminas se han descrito como recalcitrantes a las bacterias anaerobias 
con excepción de algunas aminas sustituidas con grupos hidroxilo y carboxilo que fueron degradadas bajo condiciones metanogénicas (Razo et al., 1997).

Las aminas aromáticas son degradadas aeróbicamente a $\mathrm{NO}_{3}^{-}, \mathrm{N}_{2}, \mathrm{CO}_{2}, \mathrm{H}_{2} \mathrm{O}$ y biomasa y sus características carcinogénicas y mutagénicas son eliminadas (O'Neill et al., 1999; Melgoza et al., 2004), por lo que la combinación de un tratamiento anaerobio para el rompimiento reductivo del enlace azo, seguido de un tratamiento aerobio para degradar las aminas producidas puede ser un sistema efectivo para lograr la mineralización de los colorantes azo. Sin embargo, hay dos limitaciones: en condiciones anaerobias la velocidad de reducción es lenta por lo que se requieren tiempos de retención hidráulica (TRH) muy largos para alcanzar la reducción del colorante y por otra parte, el desconocimiento del destino final de las aminas aromáticas en la fase aerobia (van der Zee y Villaverde, 2005).

En la reducción de colorantes azo, la velocidad de reacción puede mejorarse por el uso de compuestos mediadores redox del tipo de las quinonas o cofactores enzimáticos como el dinucleótido de adenina flavina (DAF), que aceleran la velocidad de reacción por el transporte de equivalentes reductores entre los compuestos electrodonadores y electroaceptores (Keck et al, 1997). En trabajos realizados por van der Zee et al. (2003), concluyeron que el uso de dosis continuas de antraquinona a concentraciones catalíticas incrementaron fuertemente las eficiencias de reducción de colorantes azo y recomiendan que el mediador redox sea inmovilizado por ejemplo en carbón activado granular (CAG) debido a que presenta varios grupos activos en su superficie, incluyendo estructuras de quinonas.

El objetivo de esta investigación fue evaluar el tratamiento del colorante azo RD23 en dos reactores: uno de lecho expandido y CAG como mediador redox y otro de biomasa suspendida, para determinar que sistema presenta mejores velocidades de reducción y tiempos de retención hidráulica. También se evaluó la producción de aminas aromáticas totales (AAT) en la fase anaerobia y la degradación de éstas en la fase aerobia del proceso.

\section{MATERIALES Y MÉTODOS}

\section{Determinación experimental de la isoterma de adsorción del RD23}

Se determinó la adsorción del RD23 en el CAG (Filtrasorb 200), mediante el método estandarizado D 3860-89 (ASTM, 1990). Se colocaron diferentes dosis de carbón activado (adsorbente) en contacto con una solución de $100 \mathrm{mg} \mathrm{L}^{-1}$ de RD23 (adsorbato) durante 24 horas. Se determinó la concentración del adsorbato después del equilibrio por unidad de peso del absorbente mediante la ecuación 1. La concentración de equilibrio se determinó espectrofotométricamente a una longitud de onda máxima $\left(\lambda_{\max }\right)$ de $501 \mathrm{~nm}$.

$q_{e}=(\mathrm{Co}-\mathrm{Ce}) \mathrm{V} / \mathrm{m}$

Donde:

$\mathrm{q}_{\mathrm{e}}=$ Concentración del adsorbato después del equilibrio, $\mathrm{mg} \mathrm{g}^{-1}$

Co $=$ Concentración inicial del adsorbato, $\mathrm{mg} \mathrm{L}^{-1}$

$\mathrm{Ce}=$ Concentración final de equilibrio del adsorbato después de la adsorción, $\mathrm{mg} \mathrm{L}^{-1}$

$\mathrm{m}=$ Masa del adsorbente, $\mathrm{g}$

$V=$ Volumen del reactor, $\mathrm{L}$

Se utilizó la isoterma de adsorción de Freundlich para determinar la máxima capacidad de adsorción del RD23 en el CAG por medio de la ecuación 2 (Metcalf y Eddy, 2003):

$(x / m)=K_{f} C e^{1 / n}$ 
Donde:

$\mathrm{x} / \mathrm{m}=$ Masa del adsorbato adsorbido por unidad de masa del adsorbente, $\mathrm{mg} \mathrm{g}^{-1}$

$\mathrm{Ce}=$ Concentración de equilibrio del adsorbato en solución después de la adsorción, $\mathrm{mg} \mathrm{L}^{-1}$

$\mathrm{Kf}$ = Factor de capacidad de Freundlich, (mg adsorbato / g de CAG) (L agua / mg adsorbato) ${ }^{1 / n}$

$\mathrm{n}$ = Parámetro de intensidad de Freundlich

\section{Sistema experimental}

Se utilizaron dos reactores cilíndricos de $34 \mathrm{~cm}$ de altura y $12 \mathrm{~cm}$ de diámetro, con un volumen total de $2 \mathrm{~L}$, operados como reactores discontinuos secuenciados (SBR, por sus siglas en inglés), como se muestran en la figura 2 . El reactor $R_{1}$ operó como un rector de lecho expandido con CAG en proporción del $30 \%$ del volumen, como medio de soporte de la biomasa y como mediador redox. El reactor $\mathrm{R}_{2}$ operó como un reactor con biomasa suspendida. Los reactores se controlaron por medio de tres bombas peristálticas de velocidad variable (Master Flex, Cole-Parmer, Modelo 72200-62) y una bomba de aireación (Modelo Elite 802) conectadas a un reloj programable (Chrontrol Model XT) para el control de la carga, recirculación, descarga y aireación respectivamente. Durante la fase aerobia el aire fue difundido desde el fondo del reactor a través de un difusor poroso. La temperatura del reactor se controló a $30 \pm 1^{\circ} \mathrm{C}$, por medio de un sistema recirculador-calentador de agua (Poly Science Model 210). Se instalaron al reactor electrodos de pH y potencial de óxido-reducción (ORP).

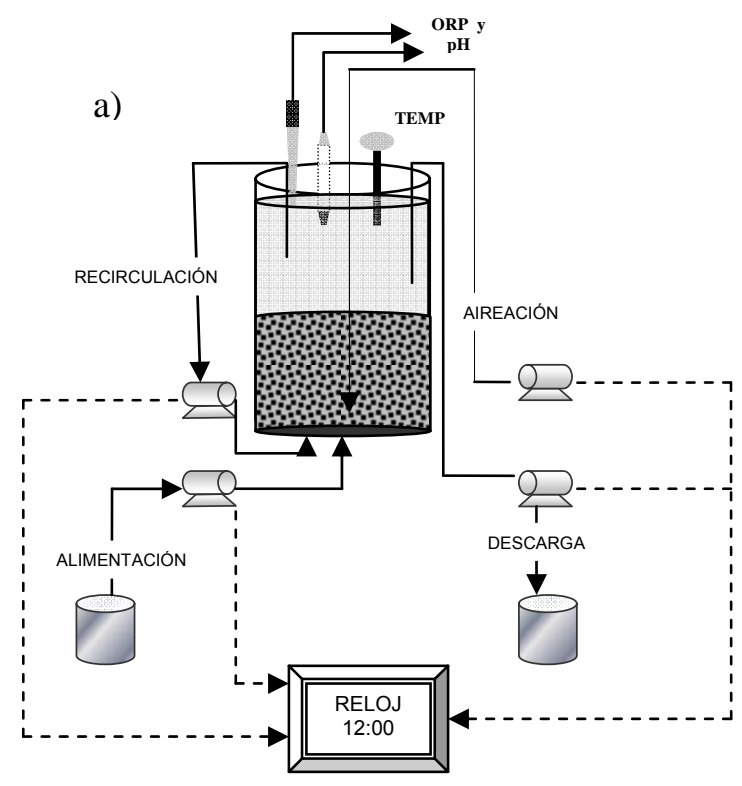

a) Reactor de lecho expandido con CAG $\left(R_{1}\right)$

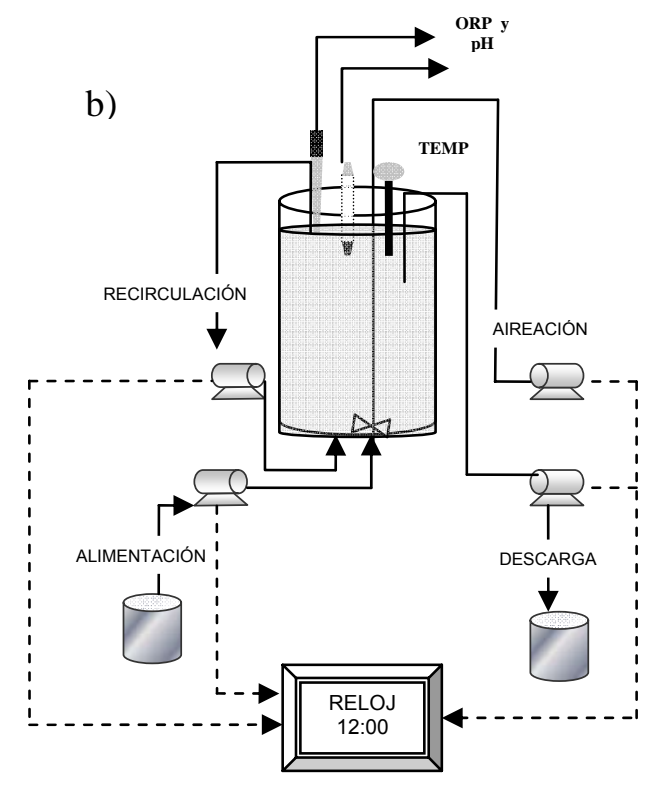

b) Reactor con biomasa suspendida $\left(R_{2}\right)$

Fig. 2: Arreglo experimental de los SBR para el tratamiento del colorante azo RD23

\section{Características del sustrato, de la biomasa y condiciones de operación}

Los reactores se inocularon con una mezcla de lodos activados aerobios provenientes de dos plantas de tratamiento, una de aguas residuales municipales y otra de aguas residuales industriales, en relación 50 / 50. La concentración de biomasa en cada reactor fue de 4,200 $\mathrm{mg} \mathrm{L}^{-1}$. Se preparó agua residual sintética con $25 \mathrm{mg} \mathrm{L}^{-1}$ de RD23, $15 \mathrm{mg} \mathrm{L}^{-1}$ de $\mathrm{CH}_{3} \mathrm{COOH}$ como cosustrato (fuente de carbono y de electrones para completar las reacciones de reducción) en relación molar 1:8 (RD23: $\mathrm{CH}_{3} \mathrm{COOH}$ ) y medio mineral nutriente con la siguiente composición (expresadas en $\mathrm{mg} \mathrm{L}^{-1}$ ). $65.25 \mathrm{~K}_{2} \mathrm{HPO}_{4}, 100.2 \mathrm{Na}_{2} \mathrm{HPO}_{4} .2 \mathrm{H}_{2} \mathrm{O}, 25.5 \mathrm{KH}_{2} \mathrm{PO}_{4}, 7.5 \mathrm{NH}_{4} \mathrm{Cl}, 22.5 \mathrm{MgSO}_{4} .7 \mathrm{H}_{2} \mathrm{O}, 27.5 \mathrm{CaCl}_{2} .2 \mathrm{H}_{2} \mathrm{O}$, $0.25 \mathrm{FeCl}_{3} .6 \mathrm{H}_{2} \mathrm{O}, 0.06 \mathrm{H}_{3} \mathrm{BO}_{3}, 0.04 \mathrm{MnSO}_{4} \mathrm{H}_{2} \mathrm{O}, 0.04 \mathrm{ZnSO}_{4} .7 \mathrm{H}_{2} \mathrm{O}, 0.03\left(\mathrm{NH}_{4}\right)_{6} \mathrm{Mo}_{7} \mathrm{O}_{24}$ y 0.1 EDTA. Para la aclimatación de la biomasa al RD23 y a los cambios de ambientes anaerobio/aerobio, en ambos reactores se utilizó la estrategia de eficiencias fijas, que consistió en permitir la adaptación de 
la biomasa el tiempo necesario hasta alcanzar el $80 \%$ de reducción del RD23 en la fase anaerobia y el $80 \%$ de eliminación de las AAT durante la fase aerobia (Melgoza et al., 2000).

\section{Métodos analíticos}

Los parámetros de $\mathrm{pH}$ y ORP se determinaron utilizando un potenciómetro (Thermo Modelo Orion $720 \mathrm{~A}+$ ). La alcalinidad total como $\mathrm{CaCO}_{3}$, fue determinada por volumetría, el oxígeno disuelto por el método electrométrico (Métodos estandarizados APHA, 2005). La determinación del colorante RD23 se realizó a la longitud de onda de $501 \mathrm{~nm}$ en un espectrofotómetro Perkin Elmer UV-VIS (Lambda 25); las AAT fueron determinadas a $440 \mathrm{~nm}$, de acuerdo al método espectrofotométrico del pdimetilaminobenzaldehído (Oren et al., 1991).

\section{RESULTADOS Y DISCUSIÓN}

\section{Adsorción del RD23 en el carbón activado}

Se realizó la determinación de la capacidad de adsorción del RD23 en el CAG para asegurar que la eliminación del colorante fuera por un proceso de biodegradación y no por adsorción. Los datos de la adsorción del colorante RD23 en el CAG mostraron una máxima capacidad de adsorción del colorante de $20 \pm 1 \mathrm{mg}$ por gramo de CAG. La isoterma de adsorción se determinó aplicando el modelo de Freundlich, debido a que es adecuado para describir la adsorción en materiales que presentan capas múltiples como el CAG (Levine, 2004) ; también se aplicó el modelo de Langmuir, pero los datos no se ajustaron a este modelo (datos no mostrados). En la figura 3 se muestran los resultados de la adsorción donde $\mathrm{q}_{\mathrm{e}}$ es la concentración de colorante en el equilibrio sobre el adsorbente y $\mathrm{Ce}$ es la concentración de equilibrio del colorante en solución; Kf y 1/n son constantes del sistema que indican la capacidad de adsorción y la intensidad de adsorción, respectivamente (Juang et al., 1997). El valor determinado para $1 / \mathrm{n}$ fue de 0.69 y Kf de 1.4312.

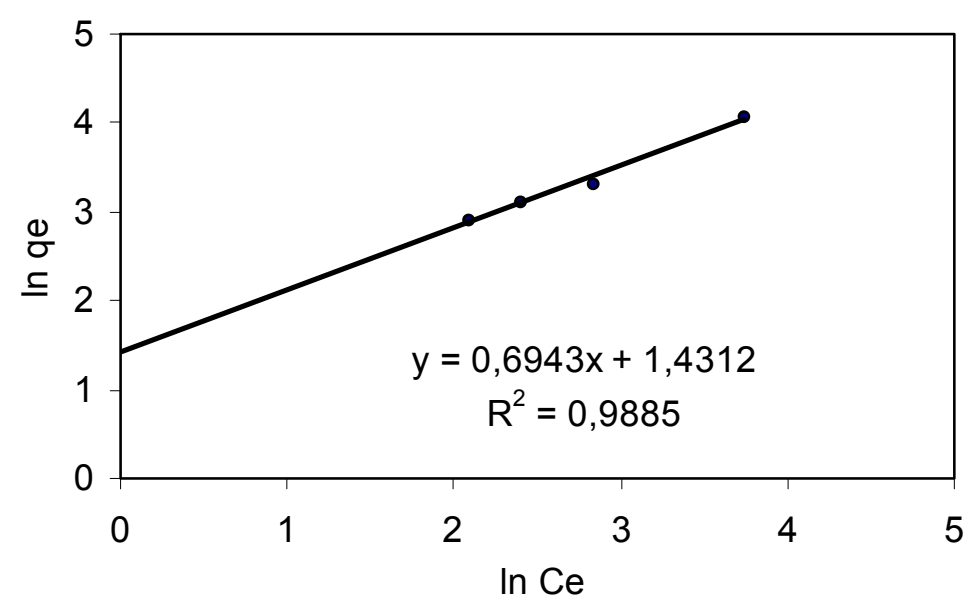

Fig. 3: Isoterma de adsorción de Freundlich para la adsorción de colorante azo RD23 en CAG (Filtrasorb 200). Ecuación de Freundlich: $\mathrm{x} / \mathrm{m}=\mathrm{K}_{\mathrm{f}} \mathrm{Ce}^{1 / \mathrm{n}}=1.4312 \mathrm{C}^{0.6943}$

\section{Aclimatación de la biomasa}

Los reactores partieron de un lodo inóculo no adaptado a la degradación del RD23, por lo que la biomasa pasó por un periodo de aclimatación incluyendo una fase de selección bacteriana utilizando ácido acético como co-sustrato para proveer electrones para la reducción del RD23, carbono y energía para el crecimiento y mantenimiento de la población bacteriana, en esta etapa el co-sustrato es consumido en su totalidad. La aclimatación de la biomasa se efectuó a través de la estrategia de operación de eficiencias de eliminación fijas. El reactor $R_{1}$ empacado con CAG operó durante 90 días y el reactor $R_{2}$ con biomasa suspendida, durante 118 días. La alimentación inicial en ambos reactores fue de $25 \mathrm{mg} \mathrm{L}^{-1}$ de RD23. La aclimatación en el $\mathrm{R}_{1}$ se alcanzó a los 60 días (ciclo 9), con 
eficiencias de degradación constantes del $83 \%$. En el $\mathrm{R}_{2}$ a los 110 días de operación (ciclo 12), la eficiencia de eliminación del colorante fue del $63 \%$, no se alcanzó el $80 \%$ establecido en la estrategia de operación, por lo que se consideró que el reactor no se había aclimatado. La razón por la que no alcanzó el $80 \%$ de reducción del colorante puede ser explicado por que el sistema no contó con los equivalentes reductores $\left(\mathrm{H}^{+}\right)$necesarios para llevar a cabo la transferencia de electrones del cosustrato al colorante azo.

En el $R_{1}$ el tiempo de retención mejoró de 288 a $72 \mathrm{~h}$, después de 60 días de operación, y en el $\mathrm{R}_{2}$ el tiempo de retención se mantuvo constante en $96 \mathrm{~h}$, después de los 110 días de operación como se muestra en la figura 4. La disponibilidad de $\mathrm{H}^{+}$derivados de la degradación del cosustrato mejoró la velocidad de reducción del RD23 permitiendo su biotransformación a AAT en un menor TRH.

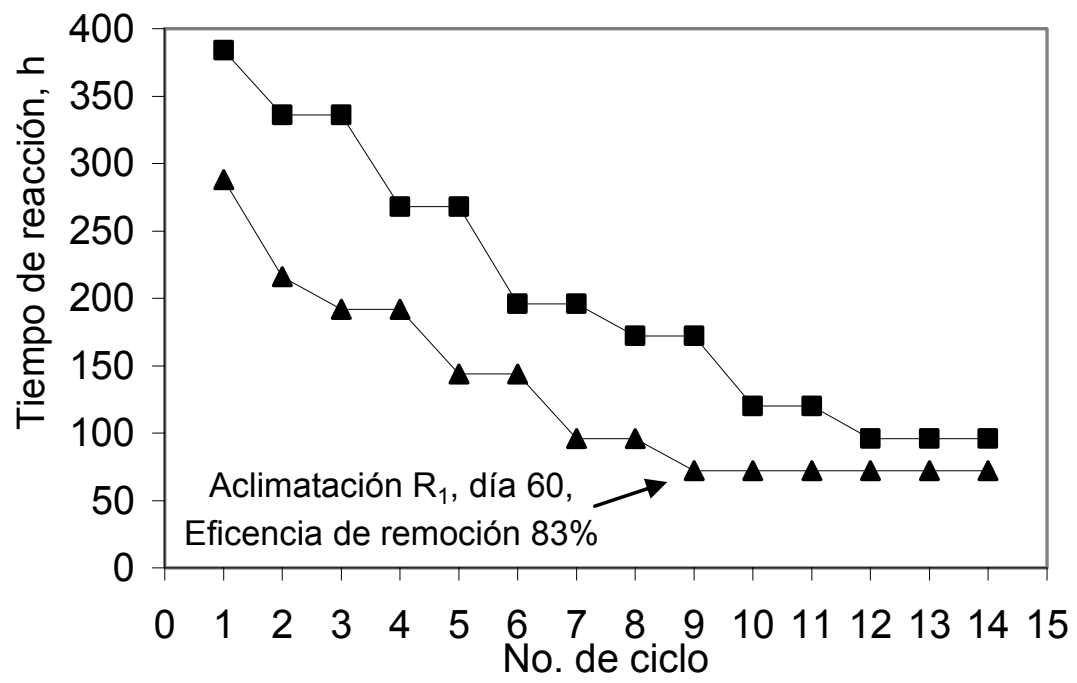

Fig. 4: Evolución del tiempo de reacción en la aclimatación de la biomasa.

(A) Reactor con CAG (匹) Reactor con biomasa suspendida

\section{Desempeño del reactor}

Después de la aclimatación, en el $\mathrm{R}_{1}$ la concentración de colorante se incrementó a $50 \mathrm{mg} \mathrm{L}^{-1}$ (ciclos 16 al 19) y a $100 \mathrm{mg} \mathrm{L}^{-1}$ (ciclos 20 al 23), con eficiencias de degradación del 84 y $90 \%$ respectivamente. La velocidad de reducción del RD23 se incrementó de 2.74 a $5.14 \mathrm{mg} \mathrm{L}^{-1} \mathrm{~h}^{-1}$ y la recuperación de aminas aromáticas totales biotransformadas fue del $8 \%$. Posteriormente en la fase aerobia del proceso las AAT fueron eliminadas por oxidación al $100 \%$. En la tabla 1 se presenta un resumen de la operación de los reactores en el proceso anaerobio/aerobio.

Tabla 1: Desempeño de los reactores en el tratamiento del colorante azo RD23 *an/ae: anaerobio/aerobio ${ }^{* *}$ AAT: Aminas aromáticas totales

\begin{tabular}{|c|c|c|c|c|c|c|c|}
\hline $\begin{array}{l}\text { TIPO DE } \\
\text { REACTOR }\end{array}$ & CICLOS & $\begin{array}{l}\text { TIEMPO } \\
\text { DE } \\
\text { REACCIÓN } \\
\text { an/ae* } \\
\text { (h) }\end{array}$ & $\begin{array}{c}\text { CONCENTRACIÓN } \\
\text { RD23 } \\
\mathrm{mg} \mathrm{L}^{-1}\end{array}$ & $\begin{array}{l}\text { EFICIENCIA } \\
\text { DE } \\
\text { REMOCIÓN } \\
\text { GLOBAL } \\
\text { RD23 } \\
(\%)\end{array}$ & $\begin{array}{c}\text { EFICIENCIA } \\
\text { DE } \\
\text { REMOCIÓN } \\
\text { AAT }^{* *} \\
(\%)\end{array}$ & $\begin{array}{l}\text { VELOCIDAD } \\
\text { DE } \\
\text { REDUCCIÓN } \\
\left(\mathrm{mg} \mathrm{L}^{-1} \mathrm{~h}^{-1}\right)\end{array}$ & $\begin{array}{c}\text { VELOCIDAD } \\
\text { DE } \\
\text { OXIDACIÓN } \\
\left(\mathrm{mg} \mathrm{L}^{-1} \mathrm{~h}^{-1}\right)\end{array}$ \\
\hline $\begin{array}{l}\mathrm{R}_{1} \text { : Lecho } \\
\text { expandido } \\
\text { con CAG }\end{array}$ & $\begin{array}{c}1-9 \\
\text { Aclimatación } \\
10-15\end{array}$ & $\begin{array}{r}288-72 \\
72-48\end{array}$ & 25 & 80 & 85 & 0.9 & 0.42 \\
\hline & $16-19$ & $48-48$ & 50 & 84 & 100 & 2.74 & 0.52 \\
\hline $\begin{array}{l}\mathrm{R}_{2} \text { : } \\
\text { biomasa } \\
\text { suspendida }\end{array}$ & $\begin{array}{c}20-23 \\
1-14\end{array}$ & $\begin{array}{r}48-24 \\
384-96\end{array}$ & $\begin{array}{l}100 \\
25\end{array}$ & 90 & 100 & 5.14 & $\begin{array}{c}0.51 \\
0.0003\end{array}$ \\
\hline
\end{tabular}


En la figura 5 se observa el desempeño del reactor de lecho expandido con CAG $\left(R_{1}\right)$. El carbón activado contiene grupos quinona en su superficie que actúan como mediador redox (Boehm, 1994), permitiendo la transferencia de electrones entre el donador (cosustrato) y el aceptor (colorante). En la fase anaerobia se observó este efecto ya que la velocidad de reducción del colorante se incrementó 2 veces más que en el $R_{2}$ al que se adicionó únicamente cosustrato. Este efecto también fue observado por van der Zee et al., (2003) quienes investigaron la importancia del carbón activado como mediador redox. La etapa limitante del proceso fue la reducción anaerobia, ya que sino se efectúa una reducción óptima del RD23 no se alcanza la biotransformación a AAT que posteriormente se oxidaran en la fase aerobia, complementando ambas fases la eliminación global del RD23.

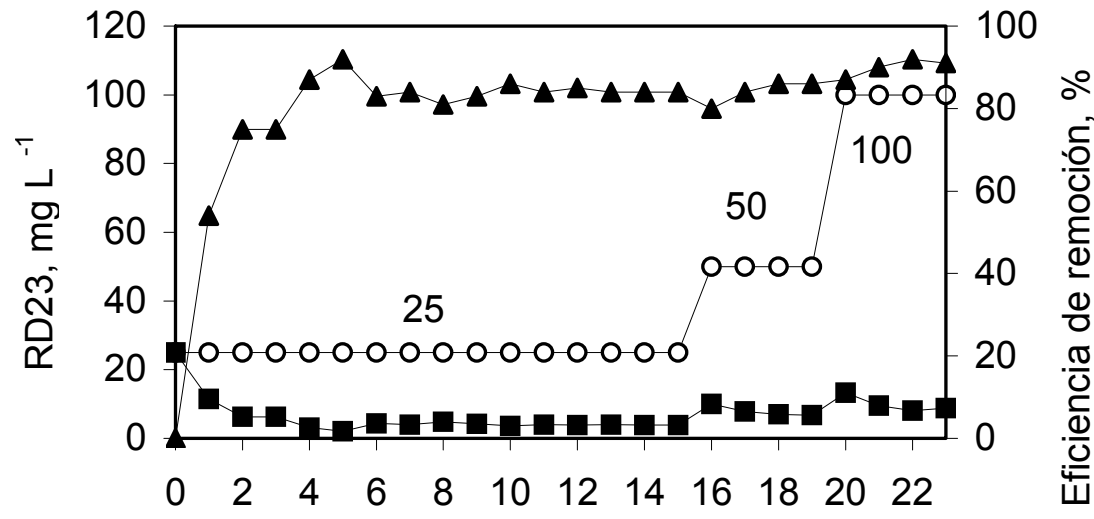

No. de ciclo

Fig. 5: Desempeño del $\mathrm{R}_{1}$ lecho expandido con CAG durante el tratamiento del RD23.

( ) RD23 inicio ciclo, (घ) RD23 final ciclo, ( $\mathbf{\Delta}$ ) Eficiencia de remoción

El proceso anaerobio/aerobio integrado en un solo reactor operado en discontinuo secuenciado, ofrece la ventaja de no requerir la purga del sistema debido a la dinámica poblacional que mantiene el equilibrio del consorcio bacteriano en la biopelícula formada, originando una baja producción de lodos. Este comportamiento también fue observado en trabajos realizados por Sirianuntapiboon et al., (2007), en el tratamiento de aguas residuales textiles en un reactor discontinuo secuenciado; Buitrón et al. (2006), van Haandel y Marais (1999), y von Sperling et al. (2001) en procesos anaerobios /aerobios para el tratamiento de aguas municipales.

Con relación a los parámetros de control, en el $\mathrm{R}_{1}$ el ORP promedio fue de $-298 \mathrm{mV}$ en la etapa anaerobia; en la etapa aerobia fue de $+114 \mathrm{mV}$ y el oxígeno disuelto se mantuvo en promedio de 5.6 $\mathrm{mg} \mathrm{L}^{-1}$. Estos resultados aseguraron condiciones de reducción y oxidación en el sistema que son condiciones indispensables en este tipo de reactores que integran ambientes anaerobios y aerobios (Kudlich et al., 1997; Rau et al., 2002). En el $R_{2}$ en la fase reductiva el ORP promedio fue de -120 $\mathrm{mV}$, no alcanzando las condiciones de reducción. El pH promedio en los reactores fue de 7.1 unidades al final de la fase anaerobia y de 8.4 unidades en la fase aerobia. Durante la fase anaerobia la alcalinidad total como $\mathrm{CaCO}_{3}$ se mantuvo en $350 \mathrm{mg} \mathrm{L}^{-1}$ que fue suficiente capacidad buffer para amortiguar los cambios de $\mathrm{pH}$. El tiempo de reacción en el $\mathrm{R}_{1}$ fue 72 horas para tratar $100 \mathrm{mg} \mathrm{L}^{-1}$ RD23, con una eficiencia de remoción del $90 \%$.

\section{Cinéticas de reacción}

Se realizaron cinéticas de reducción y biotransformación del RD23 a AAT en la etapa anaerobia y de eliminación de las AAT en la fase aerobia (figura 6). La velocidad de reducción del RD23 (qr) en el $R_{1}$ fue de $5.14 \mathrm{mg} \mathrm{L}^{-1} \mathrm{~h}^{-1}$, la velocidad de biotransformación del RD23 a AAT fue de $0.15 \mathrm{mg} \mathrm{L}^{-1} \mathrm{~h}^{-1}$ y la velocidad de eliminación de AAT fue de $0.51 \mathrm{mg} \mathrm{L}^{-1} \mathrm{~h}^{-1}, 3$ veces mas rápida que la velocidad de biotransformación. 


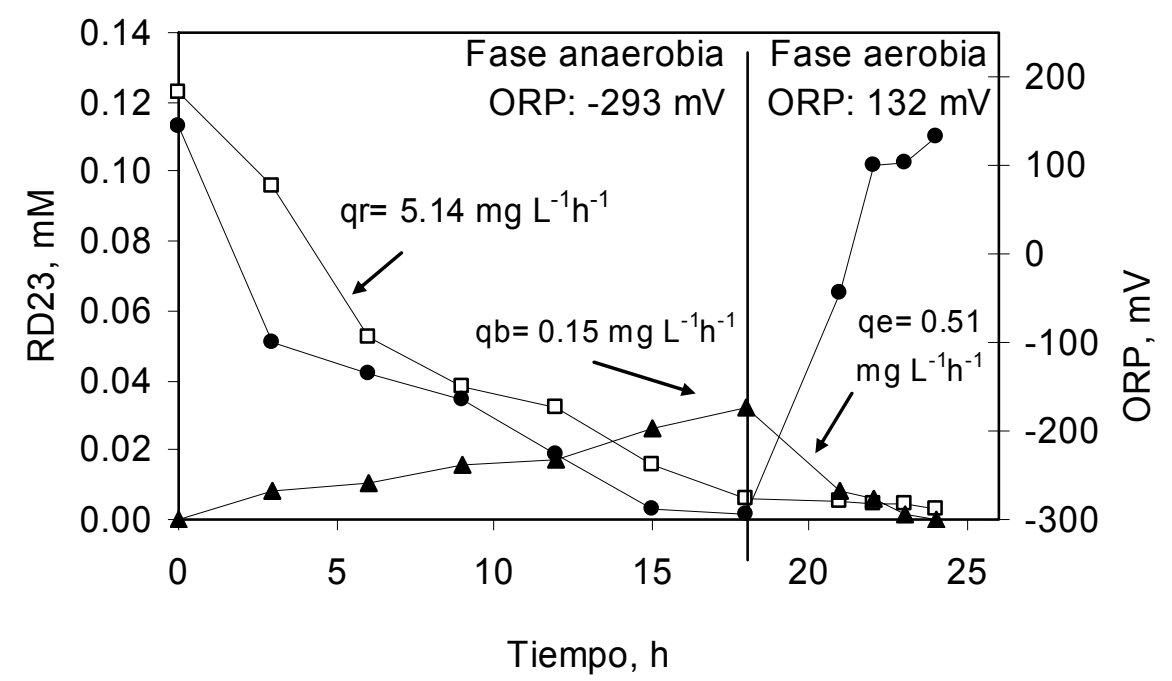

Fig. 6: Cinética de degradación del RD23 en el $\mathrm{R}_{1}$ con CAG (Ciclo 23, $100 \mathrm{mg} \mathrm{L}^{-1}(0.123 \mathrm{mM})$ $(\square)$ RD23, ( $\Delta$ ) AAT, (•) ORP

En este trabajo la adición de CAG como mediador redox en el $\mathrm{R}_{1}$ favoreció la fase de reducción del colorante, incrementando 12 veces la velocidad de reducción. Dos Santos et al. (2004), utilizaron como mediadores redox antraquinona-2,6-disulfonato, antraquinona-2-sulfonato y Riboflavina, para evaluar el efecto de éstos en la velocidad de reducción de los colorantes Rojo Reactivo 2, Naranja ácido 7 y Amarillo mordente 10 en pruebas a nivel laboratorio y observaron que con el uso de mediadores redox la velocidad de reducción se incrementó 8 veces. Sirianuntapiboon y Sansak (2007), trataron agua residual textil que contenía RD23 mediante un sistema de CAG-SBR, la concentración que evaluaron fue de $40 \mathrm{mg} \mathrm{L}^{-1}$ donde obtuvieron eficiencias de remoción del 94-97\% en un TRH de $180 \mathrm{~h}, 2.5$ veces más que el TRH alcanzado en este trabajo.

\section{CONCLUSIONES}

El tratamiento del colorante azo RD23 en el reactor de lecho expandido y carbón activado granular como mediador redox fue más eficiente para la eliminación del RD23 y de las aminas aromáticas generadas de su biotransformación.

La eliminación del colorante fue por microorganismos y no por adsorción en el carbón activado. La capacidad de adsorción del RD23 en el carbón activado fue de $20 \mathrm{mg} \mathrm{g}^{-1}$.

El carbón activado actuó como mediador redox favoreciendo la velocidad de reducción del colorante 12 veces más.

La aclimatación del reactor de lecho expandido se alcanzó a los 60 días de operación, ciclo 9 . El tiempo de retención se optimizó de 288 h a 72 h. La concentración del RD23 se incrementó de 25 a $100 \mathrm{mg} \mathrm{L}^{-1}$ con eficiencias de eliminación global promedio del $90 \%$.

La máxima velocidad de reducción fue de $5.14 \mathrm{mg} \mathrm{L}^{-1} \mathrm{~h}^{-1}$. En la fase anaerobia la recuperación de aminas aromáticas totales fue del $8 \%$, éstas fueron eliminadas al $100 \%$ en la fase aerobia.

Para contrastar la eficacia del tratamiento propuesto en este trabajo se requieren posteriores estudios en sistemas en continuo y a mayor escala.

\section{AGRADECIMIENTOS}

A la Facultad de Ciencias Químicas e Ingeniería de la Universidad Autónoma del Estado de Morelos (México) y al Consejo Nacional de Ciencia y Tecnología, por el apoyo otorgado. 


\section{REFERENCIAS}

APHA; Standard Methods for the Examination of Water and Wastewater, 21st ed. American Public Health Association/American Water Works Association/Water Environment Federation, Washington D.C: USA (2005).

ASTM D 3860-89a; Standard practice for determination of adsorptive capacity of activated carbon by aqueous phase isotherm technique. (1990).

Boehm, H. P.; Some aspect of the surface chemistry of carbon blacks and other carbons; Carbon: 32 , 759-769 (1994).

Buitrón G., E. Razo-Flores, M. Meraz y F. Alatriste-Mondragón; Biological wastewater treatment systems. Advanced biological treatment processes for industrial wastewaters. IWA Publishing: 141180 (2006).

C.I.; Colour Index. Society of Dyers and Colourists and the American Association of Textile Chemists and Colourists: $3^{\text {rd }}$ edition, Volume $9 / 5$ (1992).

Dos Santos, A.B., I.A.E. Bisschops, F.J. Cervantes y J.B. Van Lier; Effect of different redox mediators during thermophilic azo dye reduction by anaerobic granular sludge and comparative study between mesophilic $\left(30^{\circ} \mathrm{C}\right)$ and thermophilic $\left(55^{\circ} \mathrm{C}\right)$ treatments for decolourisation of textile wastewaters. Chemosphere: 55, 1149-1157 (2004).

Dos Santos, A.B., F.J. Cervantes y J.L. Van Lier; Review paper on current technologies for decolourisation of textile wastewaters: Perspectives for anaerobic biotechnology. Bioresource Technology: 98, 2369-2385 (2007).

Ekici, P., G. Leupold, y H. Parlar; Degradability of selected azo dye metabolites in activated sludge systems. Chemosphere: 44, 721-728 (2001).

Frijters R.H., R.H. Vos, G. Scheffer y R. Mulder; Decolorizing and detoxifying textile wastewater, containing both soluble and insoluble dyes, in a full scale combined anaerobic/aerobic system. Water Research: 40, 1249-1257 (2006).

Isik, M. y D.T. Sponza; Fate and toxicity of azo dye metabolites under batch long-term anaerobic incubations. Enzyme and Microbial Technology: 40, 934-939 (2007).

Juang, R.S., R.L. Tseng, F.C. Wu y H.S. Lee; Adsorption behavior of reactive dyes from aqueous streams onto chitosan. J. Chem. Technology Biotechnol: 70 (4), 391-399 (1997).

Keck, A. y otros cinco autores; Reduction of azo dyes by redox mediators originating in the naphtakenesulfonic acid degradation pathway of Sphingomonas $s p$. Strain BN6. Applied Environmental Microbiology: 63, 3684-3690 (1997).

Kudlich, M., A. Keck, J. Klein, y A. Stolz; Localization of the enzyme system involves in anaerobic reduction of azo dyes by Sphingomonas sp. Strain BN6 and effect of artificial redox mediators on the rate of azo dye reduction. Applied Environmental Microbiology: 63, 3691-3694 (1997).

Levine, I. N.; Fisicoquímica, $5^{\text {a }}$ edición, Volumen 1, 485-489. McGraw Hill, Madrid, España (2004).

Melgoza, R.M., M. Chew, y G. Buitrón; Start-up of a sequential anaerobic/aerobic batch reactor for the mineralization of p-nitrophenol. Water Science and Technology: 42(5-6), 289-292 (2000). 
Melgoza, R.M., A. Cruz y G. Buitrón; Anaerobic/Aerobic Treatment of Colorants Present in Textile Effluents. Water Science and Technology: 50(2), 149-155 (2004).

Metcalf and Eddy, Inc; Wastewater Engineering Treatment and Reuse: Fourth edition. Mc Graw Hill International Edition: 314-324 (2003).

O'Neill, C. y otros cuatro autores; Anaerobic and aerobic of simulated textile effluent, Journal Chemical Technology and Biotechnology: 74, 993-999 (1999).

Oren, A, P. Gurevich y Y. Henis; Reduction of Nitro substituted aromatic compound by the Eurobacteria Haloanaerobium pravalens and Sporohalobacter marismortium. Appl Environmental Microbiol: 57(11), 3368-70 (1991).

Rau, J., H.J. Knackmuss y A. Stolz; Effects of different quinoid redox mediators on the anaerobic reduction of azo dyes by bacteria. Environmental Science and Technology: 36, 1497-1504 (2002).

Razo, F.E., y otros cuatro autores; Complete biodegradation of azo dye azodisalicylate under anaerobic conditions. Environment Science and Technology: 31, 2098-2103 (1997).

Shaul, G.M., T.J. Holdsworth, C.R. Dempsey y K.A. Dostal; Fate of water soluble azo dyes in the activated sludge process. Chemosphere: 22, 107-119 (1991).

Sirianuntapiboon S., O. Sadahiro y P. Salee; Some properties of a granular activated carbonsequencing batch reactor (GAC-SBR) system for treatment of textile wastewater containing direct dyes. Journal of Environmental Management: 85, 162-170 (2007).

Sirianuntapiboon S. y J. Sansak; Treatability studies with granular activated carbon (GAC) and Sequencing Batch Reactor (SBR) system for textile wastewater containing direct dyes. Journal of Hazardous Materials: Accepted manuscript (2007).

Stolz, A.; Basic and applied aspects in the microbial degradation of azo dyes. Applied Microbiology Biotechnology: 56, 69-80 (2001).

Tan, N.C G. y cuatro autores mas; Degradation of azo mordant yellow 10 in a sequential anaerobic and bioaugmented aerobic bioreactor. Water Science and Technology: 42, 5-6, 337-334 (2000).

Van Haandel, A. y G. Marais; O Comportamento do sistema de lodo ativado. Teoría e aplicações para proyectos e operação. Universidade Federal de Paraiba, Campina Grande Brazil pp 88 (1999).

Van der Zee, F.P., I.A.E. Bisschops, G. Lettinga, J. A. Field; Activated carbon as an electron acceptor and redox mediator during the anaerobic biotransformation of azo dyes. Environmental Science and Technology: 37, 402-408 (2003).

Van der Zee, F.P. y S. Villaverde; Combined anaerobic-aerobic treatment of azo dyes.-A short review of bioreactor studies. Water Research: 39, 1425-1440 (2005).

Von Sperling, M., V.H. Freire, y C.A.L. Chernicharo; Performance evaluation of a UASB-activated sludge system treating municipal wastewater. Water Science and Technology: 43 (11), 323-328 (2001).

Weisburger, J.H.; Comments on the history and important of aromatic and heterocyclic amines in public health. Mutat. Research: 506-507, 9-20 (2002). 